\title{
Observation of interacting Josephson vortex chains by magnetic force microscopy
}

\author{
Sergey Yu. Grebenchuk $\odot,{ }^{1}$ Razmik A. Hovhannisyan $\odot,{ }^{1}$ Viacheslav V. Dremov $\odot,{ }^{1,2}$ Andrey G. Shishkin $\odot,{ }^{1,2}$ \\ Vladimir I. Chichkov, ${ }^{3}$ Alexander A. Golubov, ${ }^{1,4}$ Dimitri Roditchev, ${ }^{5,1}$ Vladimir M. Krasnov ${ }^{\circ},{ }^{6,1, *}$ \\ and Vasily S. Stolyarov (1,2,3,† \\ ${ }^{1}$ Moscow Institute of Physics and Technology, 141700 Dolgoprudny, Russia \\ ${ }^{2}$ Dukhov Research Institute of Automatics (VNIIA), Sushchevskaya 22, Moscow 127055, Russia \\ ${ }^{3}$ National University of Science and Technology MISIS, 119049, Moscow, Russia \\ ${ }^{4}$ Faculty of Science and Technology and MESA+ Institute of Nanotechnology, 7500 AE Enschede, The Netherlands \\ ${ }^{5}$ Laboratoire de Physique et d'Etudes des Materiaux, LPEM, UMR-8213, ESPCI-Paris, PSL, CNRS, Sorbonne University, 75005 Paris, France \\ ${ }^{6}$ Department of Physics, Stockholm University, AlbaNova University Center, SE-10691 Stockholm, Sweden
}

(Received 15 February 2020; accepted 7 April 2020; published 29 April 2020)

\begin{abstract}
The ability to control Josephson vortices is instrumental for development of superconducting cryoelectronics. However, direct visualization of multivortex states in Josephson junctions is a challenging task. Here, we employ a magnetic force microscopy (MFM) for the analysis of planar Josephson junctions. We observe a specific MFM response, seen as a chain of small rings. By changing the applied field, we show that the number of rings is equal to the number of flux quanta in the junction. Therefore, each ring represents an individual vortex in a one-dimensional vortex chain within the junction. Our observation demonstrates that the MFM technique can be used for visualization of Josephson vortices and for probing their spatial configurations and mutual interaction.
\end{abstract}

DOI: 10.1103/PhysRevResearch.2.023105

Abrikosov and Josephson vortices (AVs and JVs) represent topological objects in superconductors and Josephson junctions (JJs), respectively. Both carry a flux quantum $\Phi_{0}$ and have a $2 \pi$-phase rotation. Yet, they are significantly different. Qualitatively, unlike AVs, JVs are usually coreless, elongated along the JJ objects, forming a one-dimensional JV chain in a multivortex state. Quantitatively, the magnetic size of the $\mathrm{AV}$ is determined by the short London penetration depth $\lambda_{L} \sim 100 \mathrm{~nm}$, whereas the JV is characterized by a much longer Josephson penetration depth $\lambda_{J} \sim \mu \mathrm{m}$. The small $\lambda_{L}$ leads to large magnetic signatures of AVs: the maximal field $B(0) \gtrsim 100$ Oe and the field gradient $\partial B / \partial r \gtrsim 10^{7} \mathrm{Oe} / \mathrm{cm}$. This leads to a relative easiness of observation of AVs, which are very well studied, visualized, and even monitored in real time using a variety of techniques: decoration [1-3], magnetooptics [4,5], scanning tunneling microscopy [6-8], magnetic force microscopy (MFM) [9,10], Hall probe [11,12], superconducting quantum interference device (SQUID) [13-16], and Lorentz [17] microscopies. Concurrently, the large $\lambda_{J}$ makes magnetic signature of JVs much weaker and more difficult to observe. Furthermore, the small field gradient together with the coreless structure of JVs makes them almost pinningless. Therefore, it is difficult to immobilize JVs during the time needed for their visualization.

\footnotetext{
*Corresponding author: vladimir.krasnov@fysik.su.se

${ }^{\dagger}$ Corresponding author: vasiliy.stoliarov@ gmail.com

Published by the American Physical Society under the terms of the Creative Commons Attribution 4.0 International license. Further distribution of this work must maintain attribution to the author $(s)$ and the published article's title, journal citation, and DOI.
}

Several ways to probe JVs directly or indirectly has been tested so far. For example, moving JVs generate electromagnetic waves, which can be probed by scanning electron microscopy [18]. However, such images represent standing waves in the $\mathrm{JJ}$ rather than JVs. Interlayer vortices in strongly anisotropic high- $T_{c}$ cuprates were observed by the scanning SQUID microscopy [19]. Those are not regular JVs in the sense that they have a small nonlinear core with a field gradient comparable to that for the AV [20]. The nonlinear core enables a strong mutual interaction with pancake AVs, leading to formation of AV chains, observable by various techniques $[1,10,11,21]$. Another type of unconventional semi-Josephson vortices, containing cores with suppressed gaps, appear in grain-boundary [12-14] or proximity-coupled JJs [22]. Core pinning of such AV-JV vortices allows sufficient immobilization for visualization.

Recently, it was demonstrated that dynamic signatures of JVs can be observed using the MFM technique [23]. The phenomenon has much in common with scanning probe imaging of quantized charge [24,25] and flux [26,27] entrance in quantum dots and mesoscopic superconductors, respectively. The MFM response is due to induction of JV dynamics (flux flow) by the oscillating MFM tip [28] and as such does not carry information about the JV structure or spatial distribution. It is not obvious that MFM would be suitable for visualization of individual JVs because the MFM tip can easily drag the mobile JV. Thus, because of progress with the observation of semi-Josephson vortices, we established a method for the investigation of the structure, distribution, or mutual interaction of Josephson vortices.

In this paper, we employ MFM for investigation of spatial distribution and mutual interaction of Josephson vortices in planar Josephson junctions. We observe a spatial modulation 

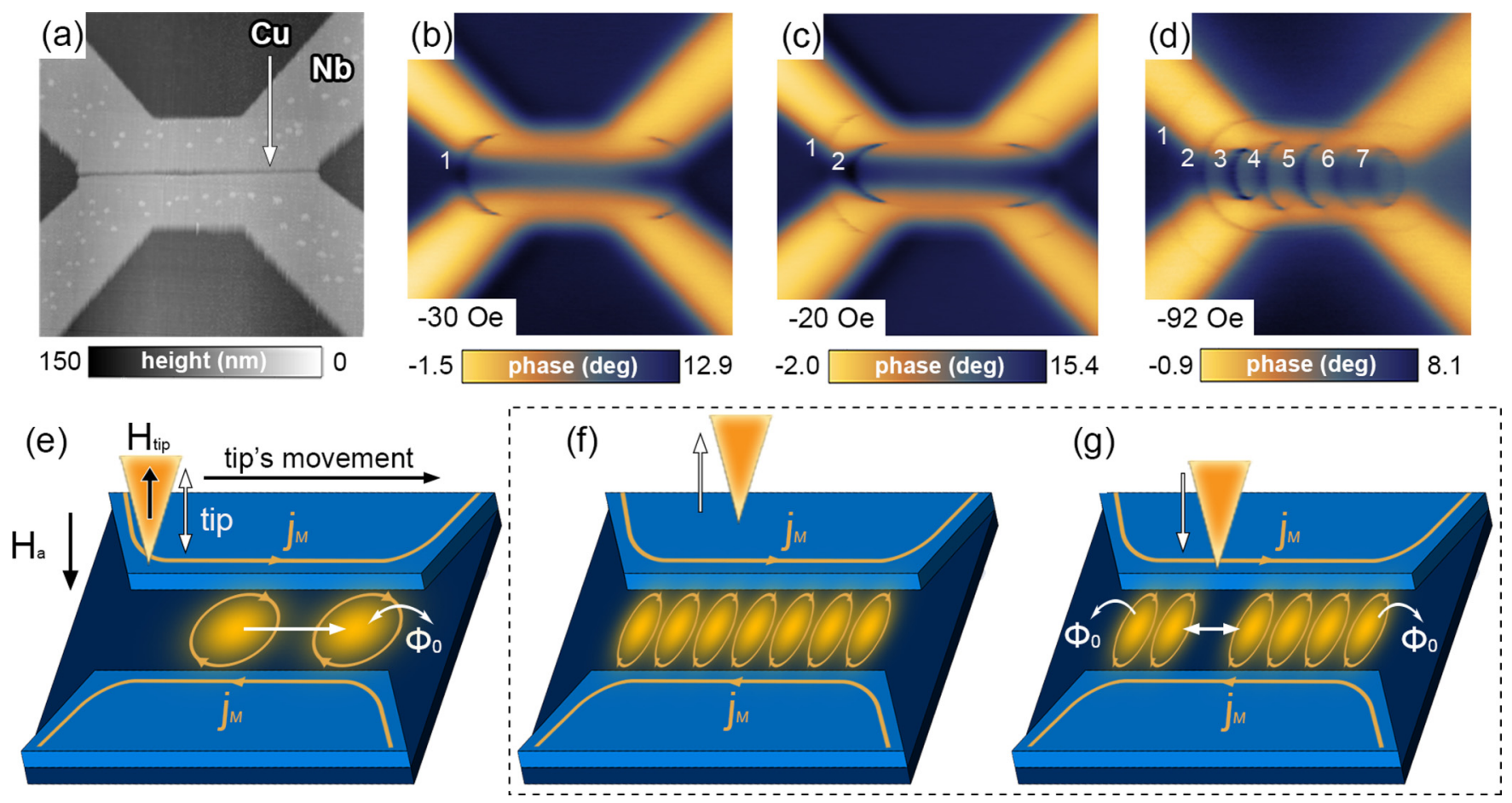

FIG. 1. Observation of dynamic Josephson vortex signatures by MFM. (a) Topographic image of a planar $\mathrm{Nb} / \mathrm{Cu} / \mathrm{Nb}$ junction. (b)(d) MFM phase maps at different applied out-of-plane magnetic fields. The scan area is $4.3 \times 4.3 \mu \mathrm{m}^{2}$. Specific MFM responses associated with Josephson vortices appear as arcs and rings in the phase maps. Their number (indicated in the maps) increases with increasing field. At high field (d), the rings form a periodic chain, reflecting static distribution of JVs in the junction. (e)-(g) Sketches of tip-induced Josephson vortex dynamics in the junction for corresponding cases shown in (c) and in (d) for the retracted tip (f) and when the tip is close to the junction (g). $H_{\text {tip }}$ corresponds to the cantilever's own magnetic field. $j_{M}$ 's show screening Meissner currents induced in superconducting $\mathrm{Nb}$ contacts.

of the MFM response, seen as a chain of small rings. By changing the magnetic field, we demonstrate that the number of rings is equal to the number of flux quanta in the JJ. Therefore, each ring represents an individual JV in the vortex chain. The visualization of JVs becomes possible due to a geometrical confinement of the elastic JV chain caused by the edge pinning [29], which, together with mutual repulsion of JVs, makes the JV chain rigid enough during MFM scans. Our observation demonstrates that the MFM can be used both for direct visualization of JV configurations and for probing their mutual interaction. We argue that the ability to monitor and control JVs could be instrumental for the development of superconducting digital electronics.

Figure 1(a) shows an atomic force microscopy (AFM) image of the studied planar $\mathrm{Nb} / \mathrm{Cu} / \mathrm{Nb} J J$. Details of fabrication can be found in Refs. [23,30]. Widths and thicknesses of the $\mathrm{Cu}$ interlayer and $\mathrm{Nb}$ electrodes are $W_{\mathrm{Cu}}=200, d_{\mathrm{Cu}}=50$, $W_{\mathrm{Nb}} \simeq 500$, and $d_{\mathrm{Nb}}=100 \mathrm{~nm}$. The $\mathrm{JJ}$ is moderately long with the length $L=2.5 \mu \mathrm{m} \sim(5-7) \lambda_{J}$. Measurements are carried out in a scanning probe system (AttoDry 1000/SU) at a temperature $T \sim 4.5 \mathrm{~K}$ in magnetic fields perpendicular to the junction plane. Both AFM, Fig. 1(a), and MFM scans are performed using a $(\mathrm{Co} / \mathrm{Cr})$-coated MFM cantilever. MFM measurements are made with the tip-sample distance of $70 \mathrm{~nm}$. The amplitude and phase of cantilever oscillations are measured at a fixed resonance frequency $\sim 87 \mathrm{kHz}$. More details about the experimental technique can be found in Ref. [23].
Figures 1(b)-1(d) represent MFM images of the JJ at different applied magnetic fields, 1 (b) $H_{a}=-30 \mathrm{Oe}$, 1(c) -20 Oe, and 1(d) -92 Oe. The MFM tip generates a positive field at the JJ, $H_{\text {Tip }} \simeq+34$ Oe. Therefore, the effective fields $H^{*}=H_{a}+H_{\text {Tip }}$ are (b) $H^{*} \simeq 4 \mathrm{Oe}$, (c) $14 \mathrm{Oe}$, and (d) -58 Oe. It can be seen that at low $H^{*}(\mathrm{~b})$, the interior of the $\mathrm{JJ}$ is empty, i.e., it does not show any significant magnetic response. However, at both JJ edges, a single dark arc appears. It corresponds to reduction of the tip phase indicating excess damping of the tip. As described in Ref. [23], the damping is caused by a periodic entrance/exit of a single JV in an oscillating field of the tip as sketched in Fig. 1(e). With increasing $\left|H^{*}\right|$, the number of arcs increases (c), and, at high fields, they form a periodic chain of rings in the interior of the $\mathrm{JJ}(\mathrm{d})$.

Figure 2 shows, in more detail, the evolution of MFM images upon variation of $H_{a}$ : (a) -34 , (b) -44 , (c) -52 , (d) -70 , (e) -80 , and (f) -92 Oe (see Supplemental Material movie1 [31]). In each panel, the upper part represents the twodimensional MFM phase map, and the lower part-the linear scan along the junction slit. Taking into account the offset by $H_{\text {Tip }} \simeq+34$ Oe, effective fields $H^{*}$ are (a) $\simeq 0$, (b) $\simeq-10$, (c) $\simeq-18$, (d) $\simeq-36$, (e) $\simeq-46$, and (f) $\simeq-58$ Oe. A sequential transformation of the phase maps with increasing $\left|H^{*}\right|$ can be seen. First, additional broad dark arcs emerge (b). Then, complex patterns appear with extra narrower arcs (c) and (d), and finally a periodic chain of small rings is established in the junction (e) and (f). Such evolution can be quantified from 

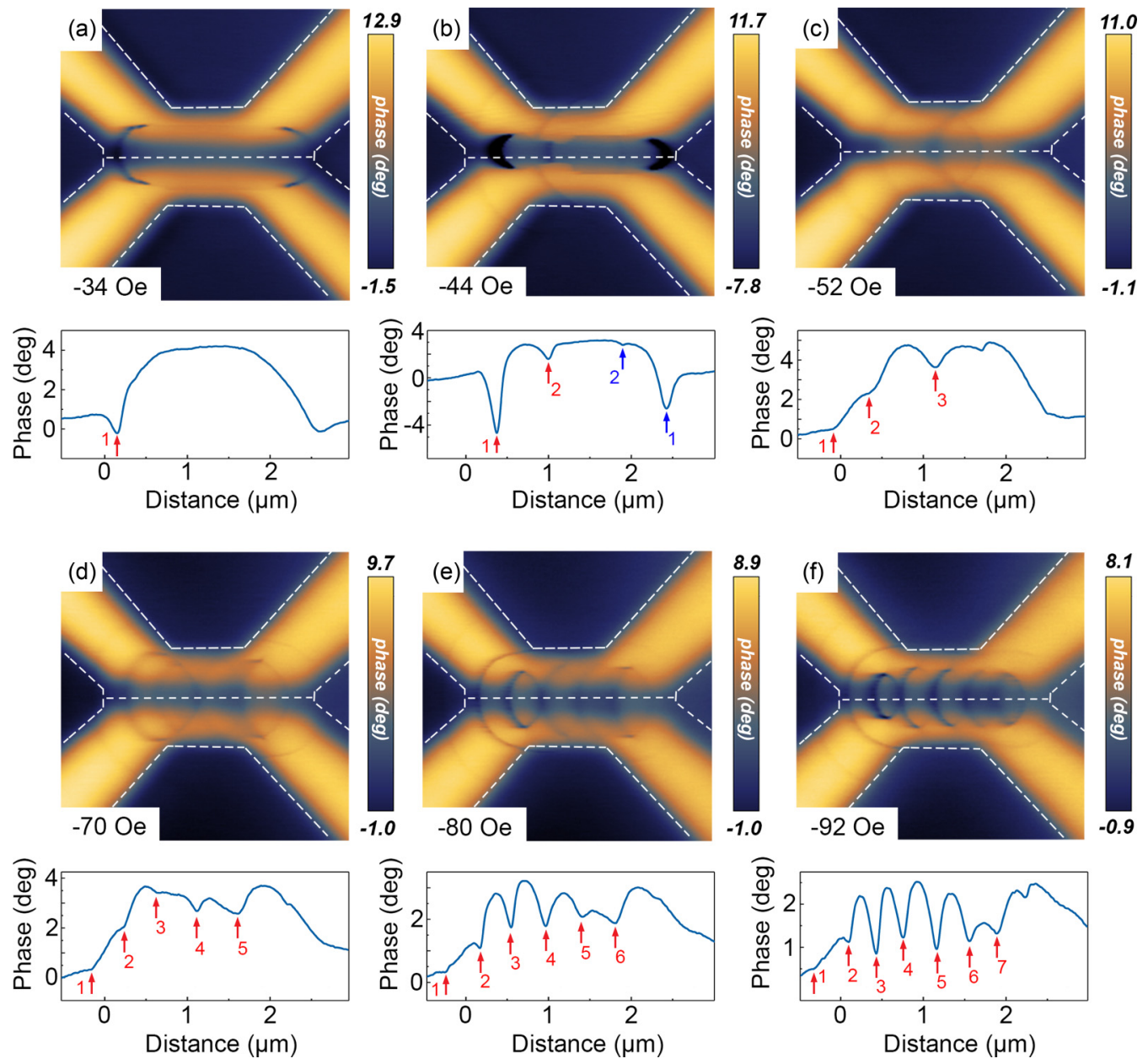

FIG. 2. Field dependence of the MFM response. (a)-(f) MFM images at different values of applied magnetic-field $H_{a}$ : (a) -34 Oe, (b) $-44 \mathrm{Oe},(\mathrm{c})-52 \mathrm{Oe},(\mathrm{d})-70 \mathrm{Oe},(\mathrm{e})-80 \mathrm{Oe}$, and (f) $-92 \mathrm{Oe}$. The top parts in each panel represent MFM phase maps with the scan area $4.3 \times 4.3 \mu \mathrm{m}^{2}$. The bottom parts represent linear scans along the junction line. It is seen that the number of arcs/rings increases with increasing $H_{a}$. The upward arrows and numbers clarify the counting procedure of the rings.

the analysis of linear scans along the junction (lower parts). Arcs/rings correspond to phase drops in the MFM signal. The gradual increase in the phase in the interior of the JJ in each scan reflects the repulsive diamagnetic response due to a partial screening in the JJ. Note that there is a clear dissimilarity of the left and right sides of the rings (f): one side is brighter than the other, which makes them look like arcs.

The increase in the number of arcs/rings correlates with the increase in the number of JVs in the junction. This is demonstrated in Fig. 3. The black dots represent the number of full rings (with both left and right arcs). The way of counting is clarified in the linear scans of Fig. 2 by red arrows and numbers. Essentially, we count only the left arc of each ring (with symmetric left and right arcs). It can be seen that the number of rings change stepwise with the field step $\sim 9.5$ Oe. The smallest number of rings is 1 , see Fig. 2(a). We do not see zero because the MFM tip field is strong enough to induce, at least, one $\Phi_{0}$ in the JJ. One ring exists in the field range of $-44 \mathrm{Oe}<H<-24 \mathrm{Oe}$, which is approximately twice the field step size. At $H>-24$ Oe, the number of rings starts to increase. Therefore, the range of -44 Oe $<H<-24$ Oe corresponds to the Meissner state of the JJ $\Phi \simeq 0$. From this, we determine the tip field $H_{\text {tip }} \simeq$ 34 Oe.

The red dots and solid lines in Fig. 3 show the estimated absolute number of flux quanta in the junction representing the equilibrium number of JVs at a given effective field. It is calculated as $\Phi / \Phi_{0}=H^{*} / \Delta H$, where $\Delta H \simeq 10 \mathrm{Oe}$ is the flux quantization field extracted from the periodicity of Fraunhofer-type modulation of the critical current versus magnetic-field $I_{c}(H)$, shown in the inset of Fig. 3. The $I_{c}(H)$ pattern is measured with a retracted tip and, therefore, does not have a field offset. From a comparison of black and red symbols, it is seen that the number of rings, indeed, correlates with the number of JVs. Therefore, each ring represents an individual JV. Moreover, the observed periodic spatial distribution of the rings, see Figs. 2(e) and 2(f), coincides with the expected spatial distribution of JVs in a one-dimensional vortex chain. Thus, the MFM technique does allow analysis of individual JVs, their structure, and spatial distribution.

The observed MFM response is caused by the tip-JV interaction. The tip creates an oscillating local magnetic field 


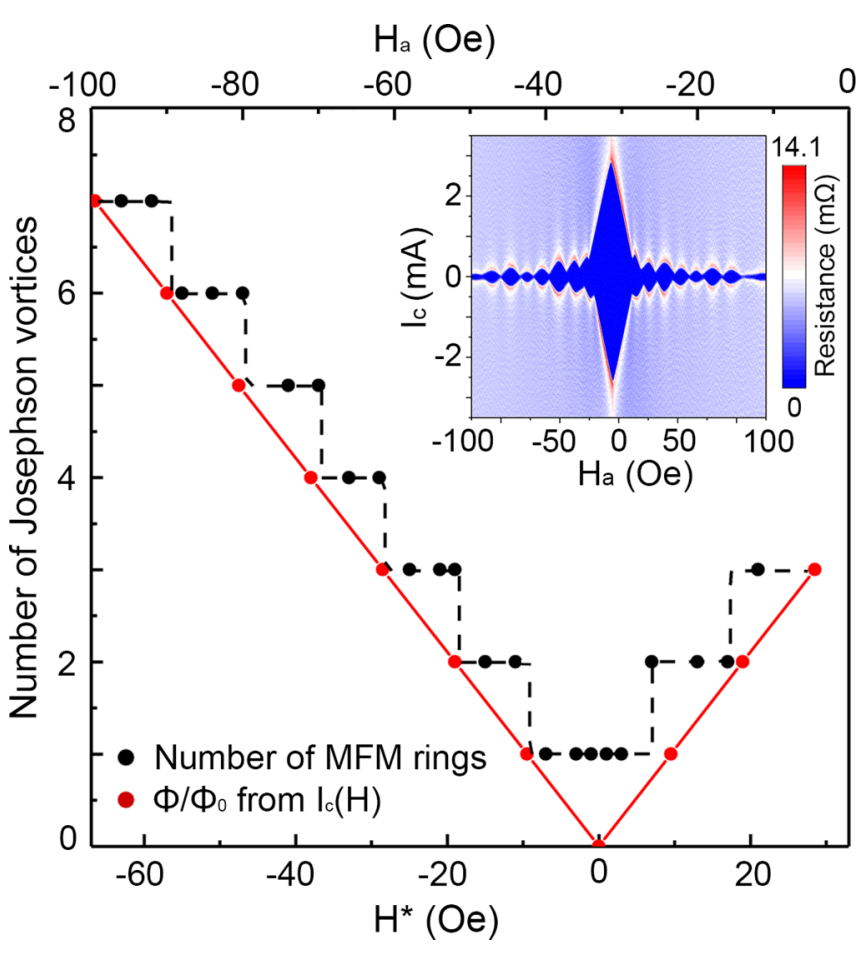

FIG. 3. Analysis of a correlation between the number of specific MFM responses and the number of Josephson vortices (flux quanta) in the junction as a function of the magnetic field. The black dots represent the number of rings (left arcs) in the MFM maps as a function of the effective field $H^{*}$ (bottom axis, offset by the tip field $H_{\text {Tip }}=34 \mathrm{Oe}$ ). The applied field $H_{a}$ is indicated in the top axis. The red symbols and lines represent the estimated number of flux quanta in the junction obtained from the periodicity of the $I_{c}(H)$ pattern. The inset shows the corresponding Fraunhofer-type $I_{c}(H)$ pattern of the junction with a retracted MFM tip.

[28]. Although it is rather small, it can, nevertheless, cause the entrance/exit of a JV at bifurcation points between $n-1$ and $n$ vortex states [23]. They occur at integer numbers of flux quanta in the $\mathrm{JJ} \Phi=n \Phi_{0}$, corresponding to minima of $I_{c}(H) \simeq 0$ and vanishingly low energy for the vortex entrance. A periodic tip-induced entrance/exit of a JV leads to the appearance of flux-flow losses that dampen tip oscillations [28]. This causes a phase drop seen as dark arcs in MFM phase maps [23]. Since JVs can only enter through the JJ edge at $\Phi \sim 0$, this type of interaction leads to the appearance of a single arc at that edge, see Fig. 1(b). The interior of the JJ remains in the Meissner state (empty). Screening Meissner currents in electrodes, marked as $j_{M}$ in Figs. 1(e)-1(g), causes a diamagnetic repulsion of the tip, corresponding to brighter areas in Fig. 1(b). With an increasing magnetic field, multiple JVs enter the junction with $N_{J V} \simeq \Phi / \Phi_{0}$.

Several forces act on JVs: They are pushed inside by the external field, repel each other, and experience attraction to the edges via interaction with image vortices [32]. The latter leads to the edge pinning. A combination of those forces leads to the formation of an elastic vortex chain, confined with junction edges [29]. Magnetic induction $B(x)$ along the chain is modulated with maxima at the vortex centra. This creates a field gradient $\partial B / \partial x$, which exerts a force on the MFM tip. If the JV chain would be rigid, the MFM scan would provide a direct image of the vortex field gradient. However, most likely, the reality is more complicated. The JV chain is soft, elastic, and only weakly pinned at the edges. Therefore, the oscillating MFM tip periodically distorts (stretches) the chain as sketched in Fig. 1(g). Due to elasticity, the distortion propagates in both directions along the chain and may lead to expulsion of the outmost JV from one of the edges. Upon retraction of the tip, the JV enters back, and the periodicity of the chain is restored. The corresponding in/out shuttling of the outmost JV leads to additional flux-flow losses, which result in damping of tip oscillations, similar to the case of tipinduced JV shuttling in the absence of the JV chain [23,28]. However, the key difference between the Meissner and the JV chain states is that the force with which the chain acts on the tip depends on the field gradient $\partial B / \partial x$ induced by the vortex chain inside the junction. Consequently, the MFM response should reflect the internal structure of the JV chain.

Since the MFM tip radius $r \simeq 30 \mathrm{~nm}$ is much smaller than the JV size $\sim 2 \lambda_{J}$, the tip-vortex force is pointlike. It can be written as $F=-m_{z} \partial B_{z} / \partial x$, where $m_{z}$ is the dipole moment of the tip and $B_{z}(x)$ is the JV field at the tip. Each JV creates a peak in $B_{z}(x)$ with two slopes on both sides at which the tip-vortex force reaches maxima. Due to opposite signs of $\partial B_{z} / \partial x$ at those points the tip pushes the JV either to one edge or the other. Mutual repulsion between JVs causes displacement of other JVs in the chain in the same direction. Consequently, each JV creates two, generally dissimilar, replicas in the dynamic MFM response (left and right arcs). They correspond to pushing of the JV chain to one of the two junction edges, eventually causing out/in motion of the outmost JV. The asymmetry of the tip position with respect to the two edges is the primary cause of dissimilarity of the left and right arcs. For example, in Figs. 1(f) and $1(\mathrm{~g})$, we sketched the situation when the tip is placed closer to the left edge of the JJ. In this case, the strains $\delta x / x$ of the JV chain segments to the left and to the right of the tip are different, simply because of different lengths of the segments. Therefore, elastic forces pushing out the outmost JVs at the left and right edges of the $\mathrm{JJ}$ (proportional to the strain) are also different, leading to the dissimilar dynamic responses. The left-right asymmetry of junction parameters (inhomogeneity) would also contribute to the dissimilarity together with the asymmetry of the tip field and shape. Most likely all those factors contribute to the observed asymmetry between left and right arcs and left and right edges of the $\mathrm{JJ}$ in our experiment Fig. 2.

To summarize, we observed a periodic chain of arcs in MFM phase maps of a planar Josephson junction. We demonstrated that the number of arcs is correlated with the number of Josephson vortices in the junction and their periodicity is consistent with the expected JV distribution in the ordered one-dimensional vortex chain formed due to mutual vortex repulsion. Therefore, we concluded that the MFM technique can reveal information about the spatial configuration of Josephson vortices and their mutual interaction. This opens an opportunity for direct visualization, manipulation, and control of Josephson vortices, which can be instrumental for development and inspection of Josephson electronic devices. 
We are grateful to I. A. Golovchansky for fruitful discussions and advice. The work was supported by the Russian Science Foundation, Grant No. 19-19-00594. The paper was accomplished during a sabbatical period of V.M.K. at the Moscow Institute of Physics and Technology supported by the 5-top-100 Program at MIPT.
[1] C. A. Bolle, P. L. Gammel, D. G. Grier, C. A. Murray, D. J. Bishop, D. B. Mitzi, and A. Kapitulnik, Observation of a Commensurate Array of Flux Chains in Tilted Flux Lattices in Bi-Sr-Ca-Cu-0 Single Crystals, Phys. Rev. Lett. 66, 112 (1991).

[2] A. Bezryadin, Yu. N. Ovchinnikov, and B. Pannetier, Nucleation of vortices inside open and blind microholes, Phys. Rev. B 53, 8553 (1996).

[3] I. V. Grigorieva, W. Escoffier, J. Richardson, L. Y. Vinnikov, and S. Dubonos, and V. Oboznov, Direct Observation of Vortex Shells and Magic Numbers in Mesoscopic Superconducting Disks, Phys. Rev. Lett. 96, 077005 (2006).

[4] P. E. Goa, H. Hauglin, Å. A. Olsen, M. Baziljevich, and T. H. Johansen, Magneto-optical imaging setup for single vortex observation, Rev. Sci. Instrum. 74, 141 (2003).

[5] I. S. Veshchunov, W. Magrini, S. V. Mironov, A. G. Godin, J.-B. Trebbia, A. I. Buzdin, Ph. Tamarat, and B. Lounis, Optical manipulation of single flux quanta, Nat. Commun. 7, 12801 (2016).

[6] A. M. Troyanovski, J. Aarts, and P. H. Kes, Collective and plastic vortex motion in superconductors at high flux densities, Nature (London) 399, 665 (1999).

[7] H. Suderow, I. Guillamon, J. G. Rodrigo, and S. Vieira, Imaging superconducting vortex cores and lattices with a scanning tunneling microscope, Supercond. Sci. Technol. 27, 063001 (2014).

[8] G. C. Ménard, S. Guissart, C. Brun, S. Pons, V. S. Stolyarov, F. Debontridder, M. V. Leclerc, E. Janod, L. Cario, D. Roditchev, P. Simon, and T. Cren, Coherent long-range magnetic bound states in a superconductor, Nat. Phys. 11, 1013 (2015).

[9] A. Volodin, K. Temst, C. Van Haesendonck, Y. Bruynseraede, M. I. Montero, and I. K. Schuller, Magnetic-force microscopy of vortices in thin niobium films: Correlation between the vortex distribution and the thickness-dependent film morphology, Europhys. Lett. 58, 582 (2002).

[10] A. Correa, F. Mompean, I. Guillamon, E. Herrera, M. GarciaHernandez, T. Yamamoto, T. Kashiwagi, K. Kadowaki, A. I. Buzdin, H. Suderow, and C. Munuera, Attractive interaction between superconducting vortices in tilted magnetic fields, Commun. Phys. 2, 31 (2019).

[11] A. Grigorenko, S. Bending, T. Tamegai, S. Ooi, and M. Henini, A one-dimensional chain state of vortex matter, Nature (London) 414, 728 (2001)

[12] B. Kalisky, J. R. Kirtley, E. A. Nowadnick, R. B. Dinner, E. Zeldov, Ariando, S. Wenderich, H. Hilgenkamp, D. M. Feldmann, and K. A. Moler, Dynamics of single vortices in grain boundaries: I-V characteristics on the femtovolt scale, Appl. Phys. Lett. 94, 202504 (2009).

[13] J. R. Kirtley, C. C. Tsuei, M. Rupp, J. Z. Sun, L. S. Yu-Jahnes, A. Gupta, M. B. Ketchen, K. A. Moler, and M. Bhushan, Direct Imaging of Integer and Half-Integer Josephson Vortices in High-Tc Grain Boundaries, Phys. Rev. Lett. 76, 1336 (1996).

[14] H. Hilgenkamp, Ariando, H.-J. H. Smilde, D. H. A. Blank, G. Rijnders, H. Rogalla, J. R. Kirtley, and C. C. Tsuei, Ordering and manipulation of the magnetic moments in large-scale superconducting $\pi$-loop arrays, Nature (London) 422, 50 (2003).

[15] D. Vasyukov, Y. Anahory, L. Embon, D. Halbertal, J. Cuppens, L. Neeman, A. Finkler, Y. Segev, Y. Myasoedov, M. L. Rappaport, M. E. Huber, and E. Zeldov, A scanning superconducting quantum interference device with single electron spin sensitivity, Nat. Nanotechnol. 8, 639 (2013).

[16] L. Embon, Y. Anahory, Ž. L. Jelic, E. O. Lachman, Y. Myasoedov, M. E. Huber, G. P. Mikitik, A. V. Silhanek, M. V. Milosevic, A. Gurevich, and E. Zeldov, Imaging of super-fast dynamics and flow instabilities of superconducting vortices, Nat. Commun. 8, 85 (2017).

[17] T. Matsuda, K. Harada, H. Kasai, O. Kamimura, and A. Tonomura, Observation of dynamic interaction of vortices with pinning centers by Lorentz microscopy, Science 271, 1393 (1996).

[18] A. Laub, T. Doderer, S. G. Lachenmann, R. P. Huebener, and V. A. Oboznov, Lorentz Contraction of Flux Quanta Observed in Experiments with Annular Josephson Tunnel Junctions, Phys. Rev. Lett. 75, 1372 (1995).

[19] K. A. Moler, J. R. Kirtley, D. G. Hinks, T. W. Li, and M. $\mathrm{Xu}$, Images of interlayer Josephson vortices in $\mathrm{Tl}_{2} \mathrm{Ba}_{2} \mathrm{CuO}_{6+\delta}$, Science 279, 1193 (1998).

[20] V. M. Krasnov, In-plane fluxon in layered superconductors with arbitrary number of layers, Phys. Rev. B 63, 064519 (2001).

[21] V. K. Vlasko-Vlasov, A. Koshelev, U. Welp, G. W. Crabtree, and K. Kadowaki, Decoration of Josephson vortices by pancake vortices in $\mathrm{Bi}_{2} \mathrm{Sr}_{2} \mathrm{CaCu}_{2} \mathrm{O}_{8+d}$, Phys. Rev. B 66, 014523 (2002).

[22] D. Roditchev, C. Brun, L. Serrier-Garcia, J. C. Cuevas, V. H. L. Bessa, M. V. Miloshevich, F. Debontridder, V. Stolyarov, and T. Cren, Direct observation of Josephson vortex cores, Nat. Phys. 11, 332 (2015).

[23] V. V. Dremov, S. Y. Grebenchuk, A. G. Shishkin, D. S. Baranov, R. A. Hovhannisyan, O. V. Skryabina, I. A. Golovchanskiy, V. I. Chichkov, C. Brun, T. Cren, V. M. Krasnov, A. A. Golubov, D. Roditchev, and V. S. Stolyarov, Local Josephson vortex generation and manipulation with a magnetic force microscope, Nat. Commun. 10, 4009 (2019).

[24] M. T. Woodside and P. L. McEuen, Scanned probe imaging of single-electron charge states in nanotube quantum dots, Science 296, 1098 (2002).

[25] J. Velasco, Jr., J. Lee, D. Wong, S. Kahn, H.-Z. Tsai, J. Costello, T. Umeda, T. Taniguchi, K. Watanabe, A. Zettl, F. Wang, and M. F. Crommie, Visualization and control of single-electron charging in bilayer graphene quantum dots, Nano Lett. 18, 5104 (2018).

[26] H. Polshyn, T. Naibert, and R. Budakian, Imaging phase slip dynamics in micron-size superconducting rings, Phys. Rev. B 97, 184501 (2018).

[27] H. Polshyn, T. R. Naibert, and R. Budakian, Manipulating multivortex states in superconducting structures, Nano Lett. 19, 5476 (2019). 
[28] V. M. Krasnov, Josephson junctions in a local inhomogeneous magnetic field, Phys. Rev. B 101, 144507 (2020).

[29] S. O. Katterwe and V. M. Krasnov, Stabilization of the in-phase fluxon state by geometrical confinement in small $\mathrm{Bi}_{2} \mathrm{Sr}_{2} \mathrm{CaCu}_{2} \mathrm{O}_{8+\delta}$ mesa structures, Phys. Rev. B 80, 020502(R) (2009).

[30] V. S. Stolyarov, T. Cren, F. Debontridder, C. Brun, I. S. Veshchunov, O. V. Skryabina, and D. Roditchev, Ex situ elaborated proximity mesoscopic structures for ultrahigh vacuum scanning tunneling spectroscopy, Appl. Phys. Lett. 104, 172604 (2014).

[31] See Supplemental Material at https://link.aps.org/supplemental/ 10.1103/PhysRevResearch.2.023105 for movie of the MFM images evolution upon variation of the external magnetic field.

[32] T. Golod, A. Pagliero, and V. M. Krasnov, Two mechanisms of Josephson phase shift generation by an Abrikosov vortex, Phys. Rev. B 100, 174511 (2019). 